\title{
Relación de los factores de riesgo y comorbilidad en el programa de prevención de caídas en usuarios hospitalizados en un hospital psiquiátrico del sector salud
}

\section{The risk and co-morbidity factors and prevention of folling in users relationship program}

EE. SusanaGallegos Sánchez • LEO. María Magdalena Aguilar Rodríguez •

LEO. María Navora Camarillo Ruíz ••

\section{Resumen}

Introducción: Los pacientes hospitalizados en una unidad de salud mental tienen el riesgo potencial de enfrentar factores de riesgo. Con la intensión de prevenir caídas en los usuarios hospitalizados Ruelas y Sarabia (10, 11), han elaborado, un modelo de valoración de los factores de riesgo como son: falta iluminación adecuada, desorden del mobiliario, falta de limpieza del área física, desconocimiento de las medidas de seguridad dentro de las instalaciones de la unidad, así como factores inherentes al paciente y su condición como son: sexo, edad, estilo de vida, movilidad, factores relacionados con el estado físico, sensoriales, estado neurológico y emocional alterados, tratamiento farmacológico y los relacionados con los dispositivos para deambular. Material y Método: Se realizó una investigación de tipo transversal y descriptiva, de Mayo del 2008 a Mayo del 2009, la muestra la conformaron 285 pacientes hospitalizados a los cuales se les aplicó una cédula de evaluación de riesgo de caídas al ingreso y cada 7 días hasta su egreso. Resultados: Durante el tiempo de hospitalización 5\% de los pacientes presentaron caída, entre las características de estos tenemos el ser mujer 76\%, recibir tratamiento con benzodiacepinas (clonazepan) 100\%, con diagnóstico de ingreso episodios depresivos graves con pensamiento o intento suicida 53\%, con conducta disruptiva, por episodio maniaco o trastorno psicótico 38\%, con reporte de resonancia magnética de pérdida de volumen de parénquima cerebral $61 \%$ y factores extrínsecos $28 \%$.

- Área de hospitalización del Instituto Nacional de Psiquiatría Ramón de la Fuente

•- Directora del Departamento de Enfermería del Instituto Nacional de Psiquiatría Ramón de la Fuente.

Dirección para correspondencia • LEO. María Navora Camarillo Ruíz: navorasan@hotmail.com

RECIBIDO: 18 DE FEBRERO DE 2009.

ENVIADO A CORRECIONES: 25 DE FEBRERO DE 2010.

ACEPTADO: 27 DE MARZO DE 2010 


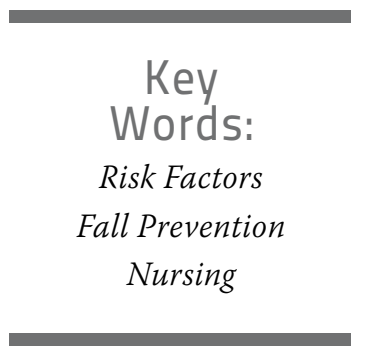

Conclusión: Se identificó que los factores de riesgo predominantes en nuestros pacientes son la combinación de psicofármacos, y la enfermedad mental, la falta de identificación del riesgo en pacientes, familiares, cuidadores y equipo de salud. En menor porcentaje influyen los factores estructurales.

\section{Key \\ Words:}

Risk Factor

Nursing
Abstract

Introduction: Hospitalized patients in a Mind Health Unit face factors of risk. Some authors $(10,11)$ have made a model to value the factors for preventing falling such as: inadequate lighting, lack of placement of furniture, lack of cleaning the physical area, lack of security measures and patient characteristics like: sex, age, life style, mobility, fitness-related factors, sensory factors, being emotional and neurological altered pharmacology treatment and related devices for wandering. Material and Methods: This is a descriptive, and transversal research realized from May 2008 to May 2009, a card falling schedule was applied to 285 patients since their income to the mind health unit and every 7 days until their discharge. Results: During the hospitalization time $5 \%$ of patients were falling; $76 \%$ were women, $100 \%$ with benzodiazepines (clonazepan) treatment, 53\% with depressing episodes and suicide attempts, 38\% with disruptive conduct for manic episode or psychotic disorder, $61 \%$ with loss brain parenchyma volume and $28 \%$ for extrinsic factors. The most frequently risk factors founded in our patients are the combination of drugs and mental illness, unknown ledge of risk by patients, their family, their careers, and health team. Structural factors risks have the lower percentage.

\section{INTRODUCCIÓN}

En el ámbito de la atención a la salud cada día es mayor el desarrollo científico y tecnológico, así mismo, cada vez son mayores las posibilidades diagnósticas y terapéuticas pero, también cada día es mayor la conciencia sobre la cantidad de situaciones que, provocadas por descuidos o errores en el sistema de salud, elevan injustificadamente el riesgo de que pacientes hospitalizados sufran daños, a veces severos e incluso fatales, con el agravante que muchos de éstos son prevenibles. El término "Seguridad del Paciente" lo define la Agencia para la Calidad y la Investigación de la Salud (Agency for Healthcare Quality and Research) ${ }^{1}$ como el "Conjunto de estructuras o procesos organiza- cionales que reducen la posibilidad de eventos adversos resultantes de la exposición al sistema de atención médica a lo largo de enfermedades y procedimientos".

Los procesos organizacionales son importantes para otorgar una atención medica segura, no obstante, contrario a lo que se piensa solo representa el 35\% de las causas de eventos adversos, por lo tanto hay otros factores. También se deben considerar los procesos organizacionales, es decir la manera en la que se otorga la atención, el modo en el que está diseñado cada proceso de atención médica para obtener el resultado deseado y cómo se entrelazan unos con otros dentro del hospital, desde que ingresa el paciente hasta que se le da su alta.

Muchas veces por falta de análisis o desconocimiento detallado de los procesos no se distingue los eslabones débiles de los que hay riesgo inminente de ocasionar un daño al paciente o al mismo personal de salud. Aquí cabe mencionar un factor fundamental: Una organización hospitalaria que no tiene el valor de la seguridad en su cultura difícilmente podrá otorgar una atención médica segura. Ha de empezar por aceptar que es falible y que todos sus miembros se pueden equivocar o cometer errores desde la más alta jerarquía hasta el paciente. Una organización hospitalaria que es capaz de darse cuenta de su vulnerabilidad, podrá 
buscar los puntos de riesgo en el proceso de atención para eliminar o prevenir, e implementar sistemas redundantes, cuando así se requiera. Lo ideal es que aprenda de sus errores, para no volver a repetirlos que diseñe procesos en donde los procesos se contengan, que todos sus miembros estén dispuestos a aceptar sus errores, darlos a conocer y hacer los cambios necesarios para que cada vez sea más segura la atención médica.

De acuerdo con lo expuesto, se deduce que la seguridad del paciente depende de varios factores. Es posible empezar por la estructura, con la que se brinda atención: dicho de otra manera, los recursos materiales, humanos y la infraestructura. Se requiere de organizaciones hospitalarias capaces de generar factores endógenos que conduzcan al cambio organizacional que la sociedad demanda, con el fin de obtener una visión macro sistémica, ya que cualquier movimiento de cada uno de sus miembros repercute de una u otra manera en el sistema, y quienes pertenecen a una organización deben saber cómo manejarse y interactuar con los diferentes equipos para alcanzar el objetivo propuesto: una atención médica segura para el paciente, los Profesionales de la Salud y para la Institución. ${ }^{1}$

Los pacientes hospitalizados con frecuencia se hayan debilitados o incapacitados, por lo que un descuido del personal de salud o el olvido de las medidas de protección pueden dar lugar a la caída del paciente. Además, muchas veces será necesario adoptar medidas adicionales con un paciente en estado alterado para prevenir que se lesiones por una caída.
Como primer paso, los miembros del personal de Enfermería deben identificar a los pacientes de alto riesgo de caer. Se recomienda hacer una clasificación de riesgo de caída de cada paciente. Luego se han de discutir brevemente las situaciones de mayor probabilidad de caída; si el paciente permanece acostado habrá que mantener elevados los barandales de la cama. También se ha de informar al enfermo y sus familiares las razones por las que se han adoptado ciertas medidas de seguridad, con el fin de evitar que alguno de ellos la interfiera o cancele. Por ejemplo, si el paciente quiere pararse, siempre tendrá que solicitar la ayuda del personal de Enfermería.

Por medio de algunas medidas sencillas se puede evitar que los enfermos sufran caídas. Los pacientes geriátricos, los pacientes que sufran algún tipo de encefalopatía u otros problemas neurológicos, los niños y los enfermos con problemas psiquiátricos merecen especial atención, lo cual ha de comunicarse a los familiares. ${ }^{2}$

La oms define a la caída, como la consecuencia de cualquier acontecimiento que precipita al paciente al suelo en contra de su voluntad. (3) La etiología de las caídas es multifactorial y por su elevada frecuencia y sus múltiples complicaciones constituyen un importante problema dentro del área hospitalaria. Existen estudios como los de Raz y Baretich de 1987 que mencionan que las caídas representan entre el 29 y $89 \%$ de todos los incidentes dentro de los hospitales. ${ }^{4}$

Existen factores intrínsecos como enfermedades crónicas, agudas y el uso de algunos fármacos o factores extrínsecos derivados de la actividad y el entorno como ilumi- nación deficiente, WC sin barras de seguridad, barandales de cama muy bajos, timbres fuera del alcance y mal estado del mobiliario., ${ }^{5,6}$ En el ámbito hospitalario los factores intrínsecos son los que influyen con mayor frecuencia en las caídas de pacientes, por lo que es necesario que la enfermera aprenda a valorar dichos factores para reducir estos eventos.

Algunos autores han elaborado un modelo de valoración de factores de riesgo para prevenir las caídas teniendo en cuenta como base el entorno, entendiéndose como el medio externo que rodea a una persona e incluye:

La iluminación adecuada reduce el riesgo de caída, sobre todo en pacientes con discapacidad visual.

El mobiliario debe de ser fuerte con sillas y sillones de respaldo altos y estables, la unidad del paciente no representara un riesgo, estará en orden y en óptimas condiciones de funcionamiento.

La limpieza del área física debe de ser impecable, sin basura ni fluidos que causen riesgos para caídas en ningún momento.

La enfermera debe de valorar el grado de conocimiento que tiene el paciente acerca de estas medidas con el objeto de orientarlo y asistirlo, mediante un plan de atención individualizado.

El estilo de vida y las dependencias provocan un riesgo adicional por el grado de estrés o ansiedad que maneja una persona, poniendo en riesgo su seguridad.

Los pacientes con limitaciones de la movilidad son más propensos a presentar caídas, ya que la inmovilidad puede predisponer a un paciente a otros accidentes y éstas pueden restringir aún más la movilidad y la dependencia. 
Factores relacionados con el estado físico y limitaciones sensoriales, los pacientes con limitaciones visuales o con limitación para la comunicación tienen elevado riesgo de lesiones, estos individuos pueden no ser capaces de percibir un riesgo potencial o expresar la necesidad de ayuda, con estos factores se ubican los pacientes con discapacidad mental, agitación psicomotriz, discapacidad física por amputaciones o malformaciones, pacientes en estado de sedación, pacientes con desnutrición, pacientes con cardiopatías, hipoxia cerebral, encefalopatías por alteraciones metabólicas, arritmias, trastornos gastrointestinales como vómito y diarrea, paresias, crisis convulsivas, deterioro auditivo, presencia de poliuria o nicturia y problemas articulares.

Los pacientes con estado neurológico y emocional alterado, tienen riesgo potencial de sufrir caídas, como los pacientes con hemiparesia parcial o total, también los que presentan ansiedad, excitación, desvalorización, tendencias suicidas, depresión, somnolencia, sopor, estupor, alteraciones de la memoria, pacientes incapaces de entender una indicación.

Los dispositivos para deambular deben de estar en óptimas condiciones, sobre todo las gomas de los bastones, muletas, andaderas y sillas de ruedas que permitan su deslizamiento fácil y frenado eficaz.

Factores relacionados con el tratamiento farmacológico; el Profesional de Enfermería debe vigilar los efectos inmediatos para extremar los cuidados que delimiten los riesgos de los siguientes fármacos.

Los hipoglucemiantes disminuyen los niveles de glucosa, lo que produce en algunos pacientes mareo y posible pérdida del equilibrio.

Los antihipertensivos, ansiolíticos, hipnóticos y sedantes entre otros pueden causar confusión, somnolencia, adinamia y cambios en la conducta.

Los betabloqueadores causan alteraciones en el sistema de conducción y posible hipotensión arterial. ${ }^{7,8,9}$

Todos los antiepilépticos causan efectos sobre el SNC en grado variable desde la somnolencia hasta las crisis convulsivas inducidas por el mismo fármaco, si las concentraciones en el plasma son lo suficientemente altas o rebasan las concentraciones terapéuticas. Los barbitúricos, las benzodiacepinas, la carbamazepina, la gabapentina, la lamotrigina, la fenitoina, las suxamidas y el ácido valproico, han sido reportados como causantes de somnolencia.

Los antidepresivos causan somnolencia, en casos aislados marcha torpe y atención alterada. ${ }^{10}$

\section{GRÁFICA 1}

Código de riesgo de caídas del paciente al ingreso a la unidad

\section{CÓDIGO DE RIESGO AL INGRESO}

ఐ VERDE BAJO RIESGO AMARILLO MEDIANO RIESGO $\square$ ROJO ALTO RIESGO

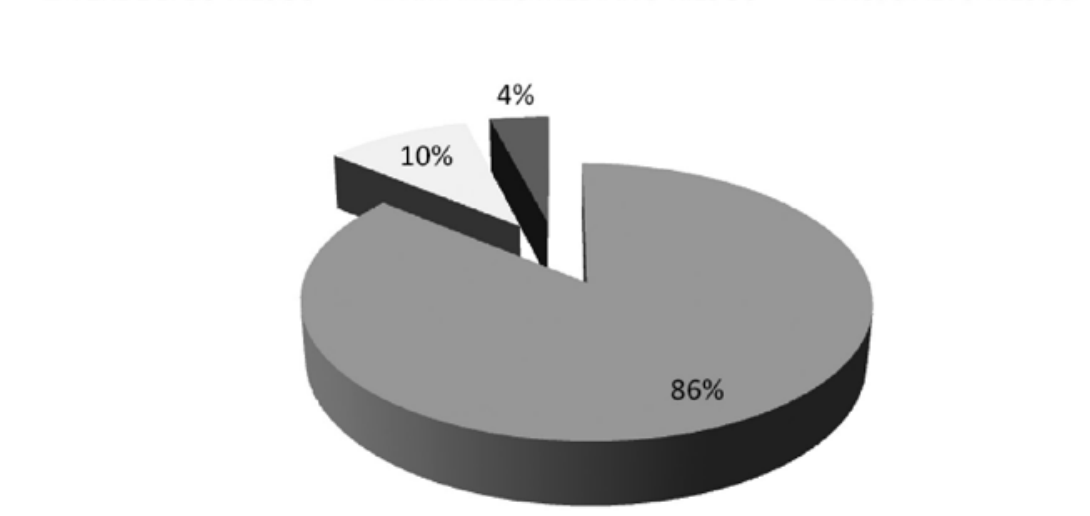

Los neurolépticos pueden producir taquicardia e hipotensión ortostatica (por el bloqueo adrenérgico). En casos severos producen síncope.

Los medicamentos antipsicóticos pueden reducir el umbral convulsivo y precipitar convulsiones gran mal.

Los efectos secundarios más comunes de las benzodiacepinas son: sedación, ataxia, deterioro cognitivo, y tendencia a causar desinhibición conductual en algunos pacientes. ${ }^{10}$

Considerando todos estos factores y experiencias relacionadas con la prevención de caídas, hablar seguridad del paciente será más que un concepto un movimiento que permita mejorar la seguridad y la calidad de la atención de los pacientes con alteraciones psiquiátricas lo cual se valora a través de la aplicación del indicador de Prevención de caídas en Pacientes Hospitalizados del sistema INDICA. 


\section{GRÁFICA 2}

Código de riesgo de caídas del paciente al egreso de la unidad

\section{CÓDIGO DE RIESGO AL EGRESO}

= CÓDIGO VERDE CÓDIGOAMARILLO = CÓDIGOROJO
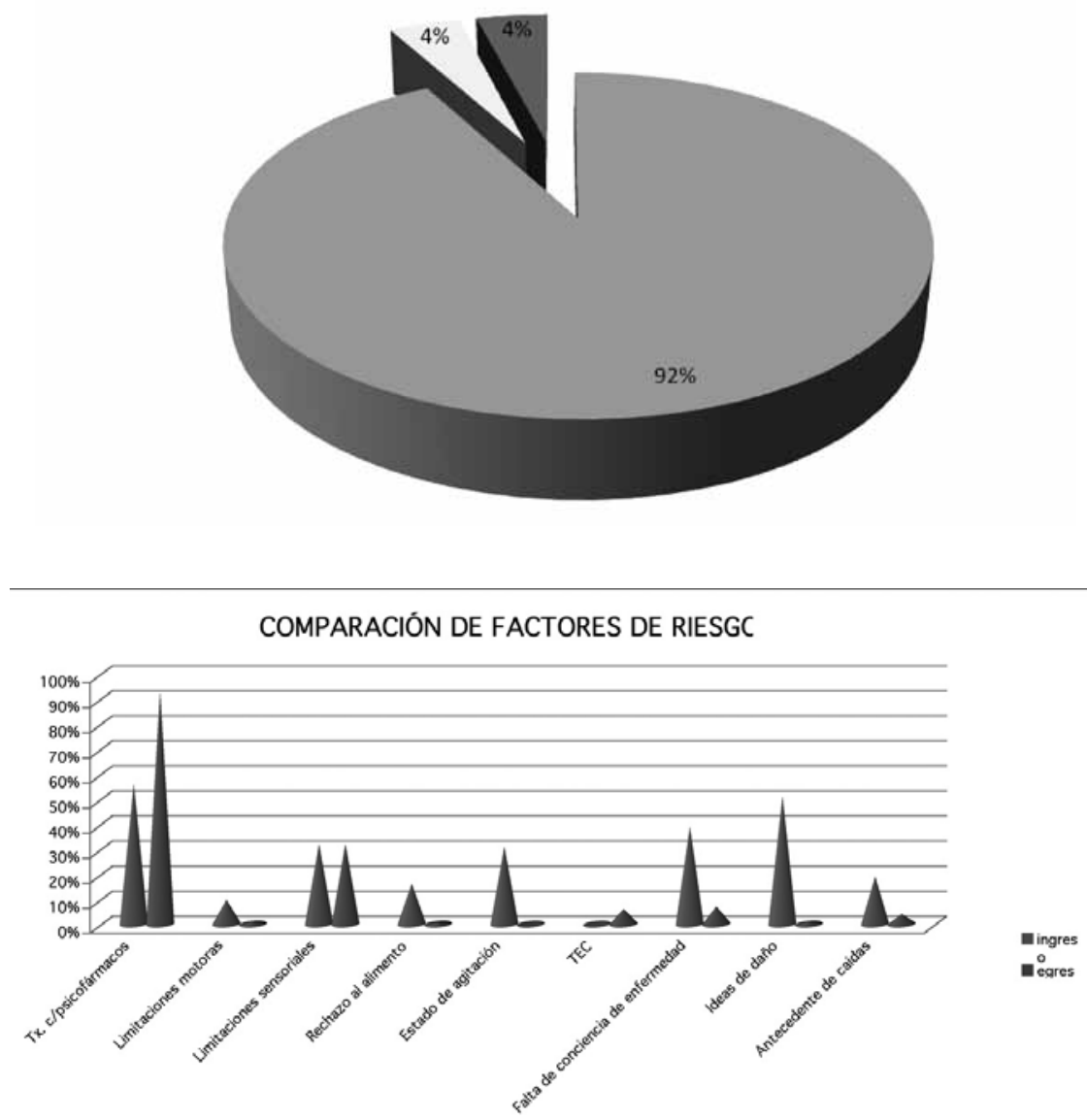

GRAFICA 3: Comparación de los factores de riesgo al ingreso y egreso del paciente a un

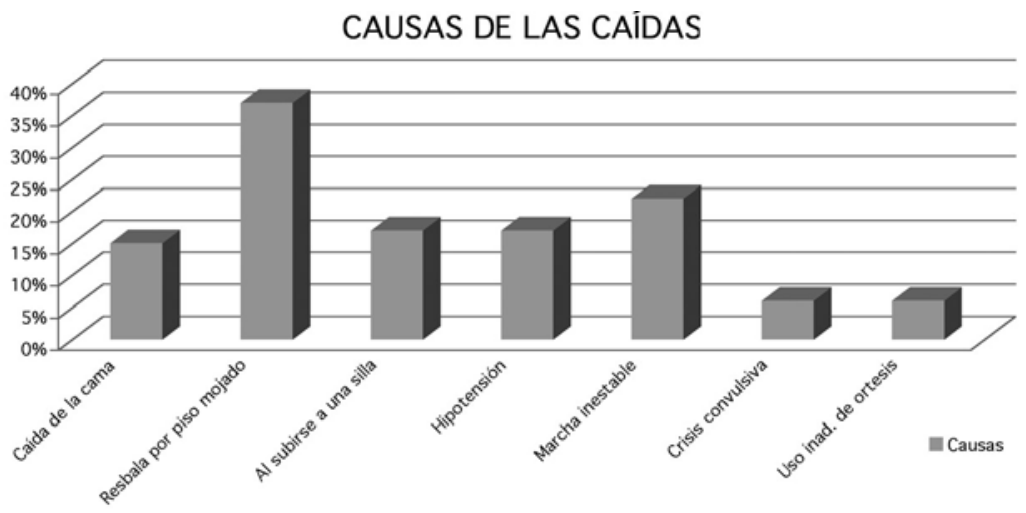

GRÁFICA 4: Frecuencia de las causas de las caídas de los pacientes

\section{Material \\ Y MÉTODO}

Se realizó una investigación descriptiva transversal, de Mayo del 2008 a Mayo del 2009. La población pacientes que ingresaron al servicio de hospitalización en el INPRFM, la muestra la conformaron 285 pacientes a los cuales se les aplicó una cédula de evaluación de riesgo de caídas al ingreso y cada 7 días hasta su egreso; participó el personal de Enfermería del Área Hospitalización para la aplicación del instrumento y se realizaron reportes de aquellos pacientes que sufrieron caídas durante su hospitalización.

Se clasificó a los usuarios desde su ingreso aplicándoles la escala de riesgo de caídas la cual consta de 12 reactivos en la que se valora capacidad sensitiva, capacidad motora, medicación con psicofármacos, la aplicación de Terapia electroconvulsiva (TEC), agitación psicomotriz, consciencia mental y neurológica, conductas agresivas, el uso adecuado de calzado y el antecedente de caídas. De esta manera se asigna a cada usuario un código de color tipo semáforo en el que los usuarios con 4 o menos puntos obtienen el código verde o que corresponde a bajo riesgo, los usuarios con 5 a 8 puntos obtienen un código amarillo o mediano riesgo y los que obtienen 9 o más puntos así como a los usuarios de 60 años y más les corresponde el código rojo o alto riesgo de caídas en este caso se aplica la escala de Tinetti para valorar marcha $y$ equilibrio.

Esta valoración se hizo cada 7 días en los que puede cambiar la condición del paciente (Cuadro 1). 


\section{RESULTADOS}

De los 285 pacientes que ingresaron al servicio de hospitalización el 76\% son del sexo femenino. (Gráfica 1). El $57 \%$ se encuentran entre 20 y 39 años de edad. (Grafica 2)

Las ideas de daño así como el uso de psicofármacos en este tipo de pacientes se consideran como los mayores factores de riesgo de caídas, las ideas de daño se reducen al recibir tratamiento sin embargo se incrementan los riesgos por los efectos secundarios del tratamiento médico. (Gráfica 3).

$\mathrm{Al}$ egreso de los usuarios el 63\% obtienen un bajo riesgo de caídas, 27 \% se mantiene con el código de ingreso, principalmente en adultos mayores quienes mantienen el código rojo durante todo en internamiento como medida de seguridad. (Gráfica 4), El uso de psicofármacos es el principal factor de riesgo tanto al ingreso como al egreso del usuario este es dado por los efectos secundarios de estos como son la somnolencia, la rigidez muscular y la hipotensión ortostatica principalmente. (Gráfica 5). Entre las causas de caídas encontramos el resbalar por el piso mojado, inquietud, la marcha inestable, la hipotensión ortostática como efecto secundario del uso de benzodiacepinas como factores principales en usuarios con agitación psicomotriz, ideas de muerte y usuarios sin conciencia de enfermedad sufrieron de caída hasta en dos ocasiones (Gráfica 6). Entre la principal sintomatológia que presentan los usuarios en relación a los Trastornos Psiquiátricos tenemos la ideación e intento suicida, los síntomas psicóticos y los trastornos de personalidad manifestados por simulación. (Gráfica 7)

\section{CUADRO CLÍNICO RELACIONADO CON LAS CAÍDAS}

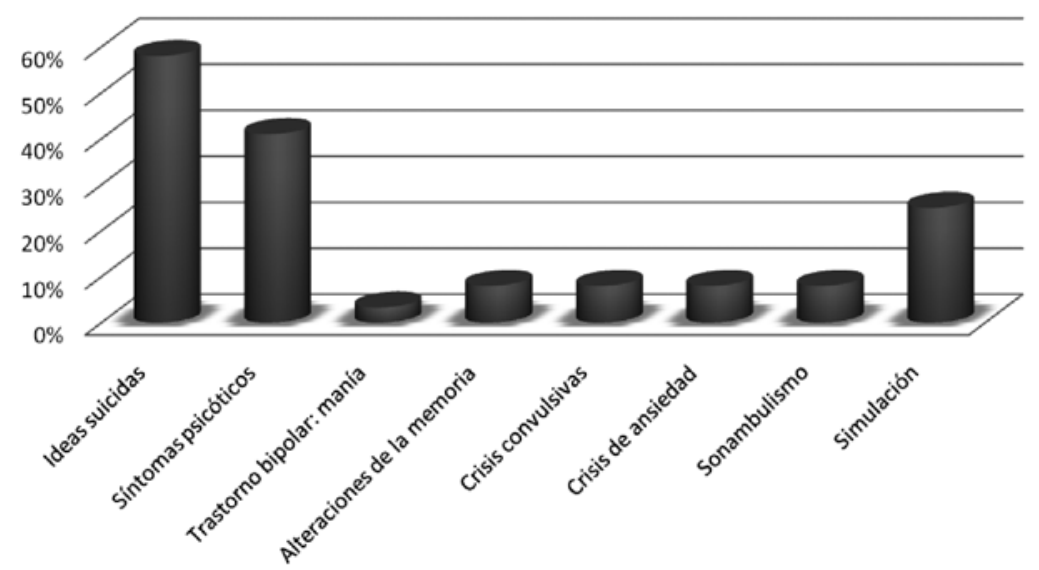

GRÁFICA: Proporción de las mejoras en el área física 2008-2009

\section{MEJORAS DEL ÁREA FÍSICA}

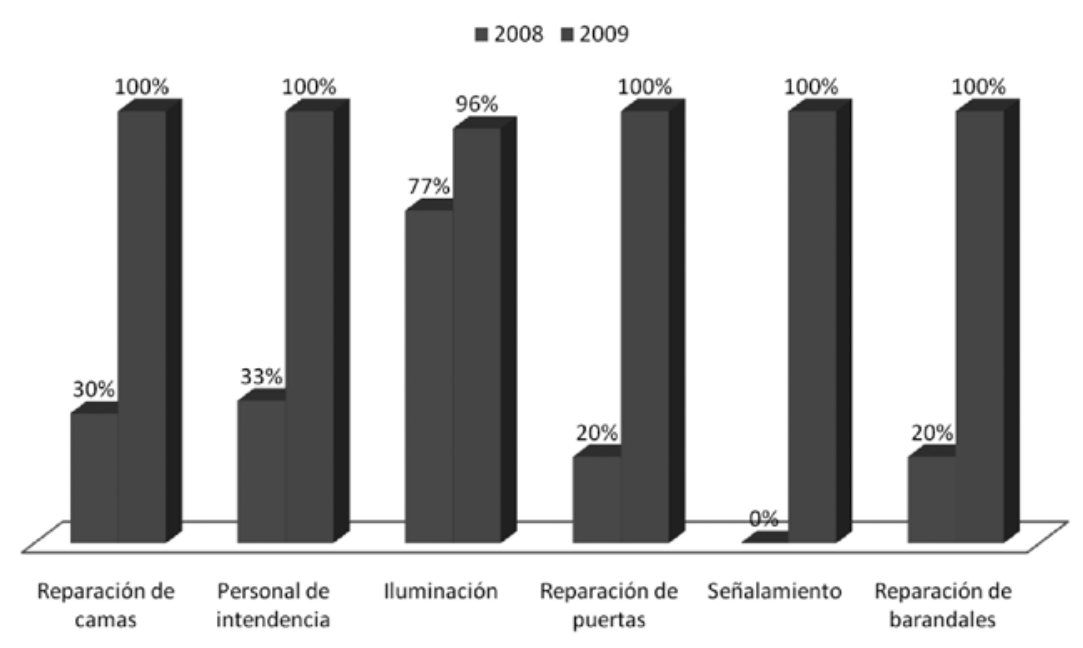

GRÁFICA: Aplicación de estrategias del programa de prevención de caídas

\section{APLICACIÓN DEL PROGRAMA}

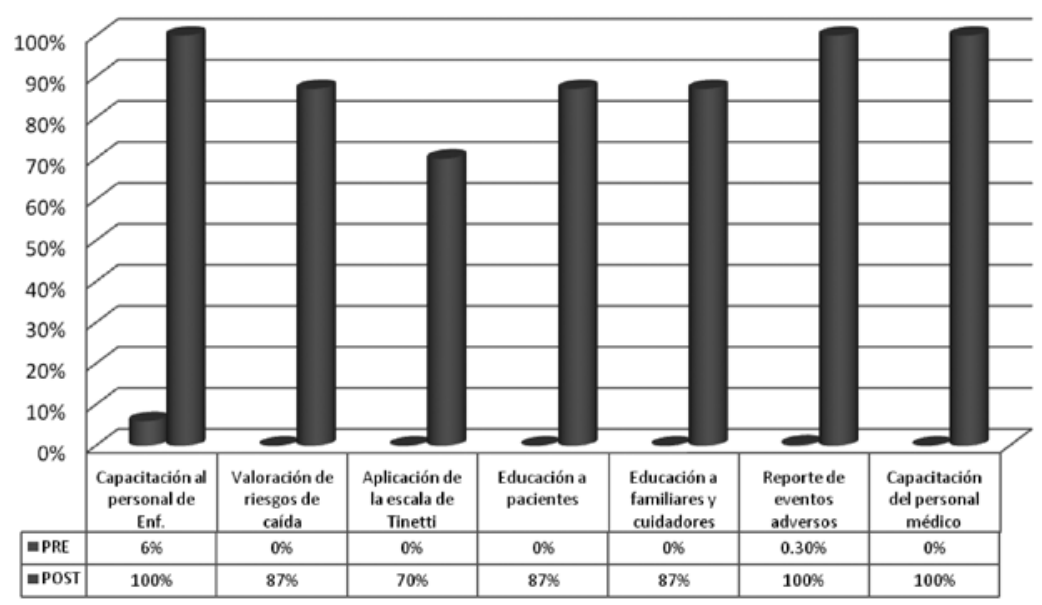




\section{DISCUSIÓN}

Se observo que la mayoría de los usuarios que fueron hospitalizados mantuvieron un código de bajo riesgo o verde a pesar de ser tratados con psicofármacos ya que el cuadro clínico que presentaban al ingreso se exacerba o minimiza, creando más conciencia sobre los factores de riesgo de caídas.

En los datos obtenidos es importante mencionar la relación de los usuarios que presentaron caídas y el uso de benzodiacepinas como parte del tratamiento, presentándose como principales efecto secundario la somnolencia y la hipotensión hortostatica, también es importante mencionar que las intervenciones de enfermería en la prevención de caídas fueron significativas, entre estas mencionamos la orientación al usuario, familia y cuidadores a su ingreso, así como reforzar la orientación al usuario durante el internamiento y como parte del plan de alta.

\section{Referencias Bibilográficas}

1. Ruelas, Sarabia Tovar. Seguridad del Paciente Hospitalizado. Instituto Nacional de Salud Pública Edit. Medica Panamericana. México: 2007

2. Comisión de Arbitraje Medico, Dirección General de difusión e investigación. Recomendaciones específicas para enfermería relacionadas con la prevención de caídas de pacientes durante la hospitalización. México: 2005

3. Navarro M y cols. Caídas del anciano en la comunidad: ¿Qué debe de hacer el médico de atención primaria? Rev. Semergen 2001;27(7): 358-361

4. Raz T, Baretich M. Factors affecting the incidente of patient falls in hospitals. Medical Care 198795-185

5. Por qué usted. Caídas. Saludalia. http://www.saludalia.com/saludalia/web_saludalia/tu_salud/doc/ anciano/doc/caídas.
6 Precauciones para evitar caídas. http://www.uam.es/centros/psicologia/paginas/cuidadores/caidas

7. Atkinson-Murray. Guía clínica para la planeación de los cuidados. Ed. McGraw-Hill Interamericana. $3^{\text {a }}$ edición México 1997: 153-183

8. Smith/Reynard. Farmacología. Ed. Panamericana. Madrid 1993. Cap. 20,21,22,33,34 y 45

9. Inestabilidad y caídas http:// web. usal.es acardoso/temas/caídas. html

10 PLM, Diccionario de especialidades, Sistema Nervioso Central, primera edición 2000 México: 126,127, 407, 408, 289-38

11. Tapia V M, Salazar C MC, Tapia CL. Olivares R C. Prevención de caídas, indicador de calidad del cuidado enfermero. Rev Mex Enferm Cardiol. 2003; 11(2): 51-57. 